\title{
Role of Electrical Forces in Angiogenesis
}

\author{
Oliver Szasz ${ }^{1}$, Gyula Peter Szigeti², Andras Szasz ${ }^{1}$, Zoltan Benyo ${ }^{2}$ \\ ${ }^{1}$ Dept. Biotechnics, St. Istvan University, Gödöllő, Hungary \\ ${ }^{2}$ Institute of Human Physiology and Clinical Experimental Research, Semmelweis University, Budapest, Hungary \\ Email: biotech@gek.szie.hu
}

How to cite this paper: Szasz, O., Szigeti, G.P., Szasz, A. and Benyo, Z. (2018) Role of Electrical Forces in Angiogenesis. Open Journal of Biophysics, 8, 49-67. https://doi.org/10.4236/ojbiphy.2018.82005

Received: January 4, 2018

Accepted: March 30, 2018

Published: April 2, 2018

Copyright (C) 2018 by authors and Scientific Research Publishing Inc. This work is licensed under the Creative Commons Attribution International License (CC BY 4.0).

http://creativecommons.org/licenses/by/4.0/

\begin{abstract}
The role of electrical forces in angiogenesis is widely studied. The electric field (EF) induces polarization of the endothelial cells, and in this way, it is a morphogen in angiogenesis. Additional to the polarization, it may build up by newborn cells in the process of cellular fission. Due to the weak direct experimental results on the transitions of endothelial cells, we used an analogy to these epithelial transitions. This involved using the injury current, which induces oriented cell migration and morphological arrangement in wound healing. The injury-current considerations are applied for malignant proliferation as well.
\end{abstract}

\section{Keywords}

Angiogenesis, Polarization, Electric Field, Injury Current, Wound Healing, Malignant Proliferation, Endothelial Cells, Epithelial Cells, Mesenchymal Cells, Cell Transitions

\section{Introduction}

Two processes are involved in the expansion of the transport network during the development of an organism: vasculogenesis and angiogenesis [1]; the first forms the rough vessel structure, while the latter is responsible for the detail, forming the network together with capillaries. In the post-developmental organism, this process is no longer active, except in special cases, when new vessel parts are required to attach to pre-existing blood vessels. The exceptions like wound healing to repair damaged tissues [2], exercise to increase oxygen delivery [3], and in pathological situations (e.g. solid tumors and inflammation) [4]. Physiological demand is required to activate angiogenesis. The natural growth of organs and muscles creates hypoxia, the elimination of which requires new vessels. The same hypoxic initialization occurs during malignant cell proliferation 
when the demand for nutrients is essential for growth.

A link between tumor growth and angiogenesis was recognized early on [5], and numerous publications have shown that angiogenesis is active in most solid tumors [6]. Tumours induce angiogenesis in the same way as the developing organ in the period before final maturation of the organism. A hypothesis was developed regarding the dependence of tumor growth and angiogenesis [7] and was subsequently experimentally proven [8]. The process is governed by the endothelial growth factor (EGF) and its specialized form, vascular endothelial growth factor (VEGF). Mature endothelial cells must allow both acute and chronic remodeling of the vessel wall.

In the first stage of angiogenesis, the form of the endothelial cells building up the vessel wall changes in a small area of the wall. The vascular tone reduces, leading to higher permeability of the wall, which could help increase the flux of nutrients in the area.

In the second stage of angiogenesis, secreted proteolytic enzymes (protease or peptidase) break the long chainlike molecules of proteins into shorter fragments, fluidizing the extracellular matrix (ECM) and promoting cellular mobility. VEGF stimulates the fission of cells, resulting in chemotaxis (chemically promoted migration) directed by the gradients of the growth factor. One of the most important aspects of this scenario is the formation of the various gradients required for morphogenesis. Two particular states exist for endothelial cells (as for other cells): interconnected (bonded) cells that form part of the tissue (endothelial cell) and autonomic, not bonded to neighboring (mesenchymal) cells. Phase-transition between the two states is possible and is called the endothelial-mesenchymal transition (EndMT). EndMT is a complex process in which endothelial cells lose their specific markers, and the adherent connections between the cells are lost when vascular endothelial cadherin (VE-cadherin) unbinds. The cytoskeleton of the cell subsequently becomes disoriented and results in deformable, autonomic mesenchymal cells, which are mobile and are capable of migrating into surrounding tissues.

EndMT was first recognized in heart development [9] [10]. A subset of endothelial cells was observed forming a primitive heart tube with a mesenchymal phenotype and invading the surrounding tissue.

Embryonic heart formation has provided most of the current knowledge about EndMT. Recently it was shown that EndMT could occur in a variety of pathological conditions including malignancies [11]. About cancer, EndMT is now recognized as a unique source of cancer-associated fibroblasts [12], facilitating tumor progression [13]. Nevertheless, the molecular mechanism of EndMT in tumors has not yet been specifically studied, although there is strong evidence that angiogenic vessels can undergo EndMT, playing a role in angiogenesis by forming tip cells, which migrate into adjacent tissue [14]. EndMT could be a major mechanism in angiogenesis [15] and could play a major role in stabilizing the neovasculature during vasculogenesis and angiogenesis. This self-organizing process 
makes the vascularization fractal, which allows a certain mathematical description of its structure [16].

\section{Cellular Transitions}

The angiogenetic vascularization and the tumor growth are well-modeled mathematically [17], but unfortunately, EndMT and angiogenesis are not understood well enough to explain the transition in detail. EndMT is often described as a form of epithelial-to-mesenchymal transition (EMT) [18]. EMT has a basic role in repairing (reconstructing) epithelial injury and can also occur in individual tumor cells as an important mechanism of invasion and metastasis [19] [20] [21] [22].

EMT has been extensively studied and offers an orienting guideline for research on EndMT. Both transitions have a similar mesenchymal phenotype and probably involve common signaling pathways. Consequently, we could use the similarities of EndMT and EMT processes, which are proceeded in some publications too, [23] [24] [25].

It is a plausible assumption that nature works by the same principles, i.e. it solves similar tasks in the same way. We call this assumption the permanence principle. On this basis, to learn about the processes taking place in endothelial cells we study the cells of the epithelium.

Epithelia separate adjacent tissues from each other and have a specific role in the homeostasis of organs, fixing the post-developed whole organism for the entire life. Epithelia are vital tissues of the body and are the basis of most complex organs. Epithelia form a well-structured layer with tight physical coupling and quasi-translational symmetry similar to crystalline materials, formed by cell-cell junctions with apical-basal polarity. The permanently polarized epithelial sheet is one of the final tissue forms of multicellular organisms.

Polarization interactions are one of the crucial factors in the building of tissues and organs [26]. They are not only decisional in epithelial cells, but active in many electrolytes in the human body, where the water structure is polarizable, semi-crystalline [27]. This ordering vanishes in malignancy [28], facilitating detection [29]. It is likely that the ordered water bound to the membrane is oriented by the membrane potential, and by the polarized epithelial sheets as well.

As the cytoskeleton defines the form of a cell, the adherent connections and junctions need a certain formal condition too. There is a stage of cells when organized in harmonic connections with their neighbors (most common are the epithelial cells) and fixed in this form, losing their intercellular chemical bonds, forming mesenchymal cells. EMT was first studied in embryogenesis. EMT, and the opposite direction the mesenchymal-epithelial transition (MET) are critical for the development of many tissues and organs in the developing embryo [30].

These processes require a change in the endothelial cells of the existing vessel in the area of attachment. Epithelial cells have an apicobasal polarity, which 
guides the polarization of the cytoskeleton. Intercellular connections (in the case of epithelial cells these typically involve E-cadherin; regarding endothelial cells these typically involve VE-cadherin) requires connected cytoskeleton inside the cells, where the cadherin-catenin-complex bonds form chains of molecules [31] (Figure 1).

Polarization is lacking in mesenchymal cells, which have a spindle-shaped morphology. While the epithelial cells can be connected chemically, the mesenchymal cells have only physical interactions (mechanical connections) with their neighbors.

EMT can induce in various ways, but the signals of the ECM have a guiding role. There are EMT effectors [32], extracellular signals [33] and collective signals [34] regulating the exchange of information between cells. This collective harmony is the basis of homeostasis of the complete organism [35]. The EMT and MET transitions may involve various chemical complexes as effectors, and in the cycle of transformations may form various endothelial structures [36] (Figure 2), developing new tissue including, unfortunately, tumorous tissues. The cells in this cycle have to break their bonds with their neighbors; they migrate to morphogenetic direction and form chains to build-up a new tube-like structure. The breakage of neighboring connections starts with the collapse of the cytoskeleton of the cells involved. During this step the cells become deformable, and the intercellular adherent connections are terminated. This is the first step in angiogenesis too, whereby the separation of the cells makes it possible to form new connected structures.

Development of the living orgasm and the consequent tissue homeostasis require the evolutionarily conserved process of EMT. EMT and MET as reverse processes, are essential for the physiological response to injury (wound healing) as well as in carcinogenesis [37] [38].

An important consequence of changes in the polarization of cells is the change in the structure of water associated with them. Water undergoes an order-disorder phase transition that may be connected with cellular proliferation [39], and

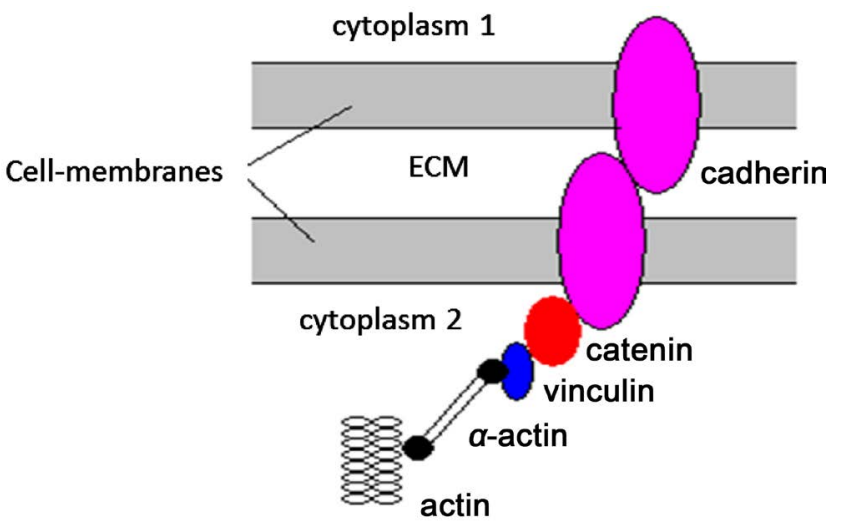

Figure 1. The adherent connection complex is one mechanism of intercellular bonding in tissues. The intercellular interactions are connected with the intracellular, affecting the actual stage of cytoskeleton. 


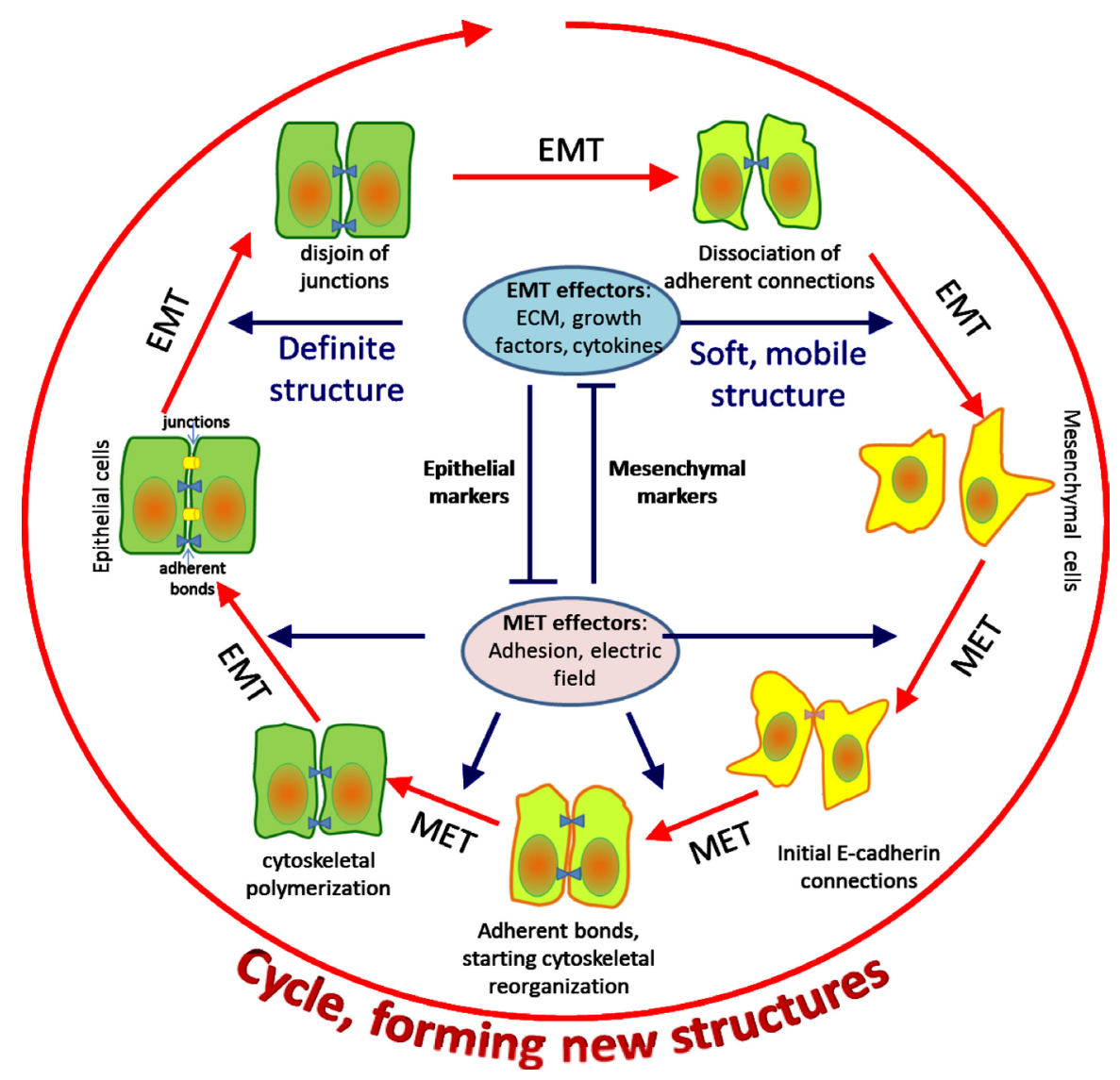

Figure 2. Interconnection of EMT-MET transformations, showing the critical effectors and the main properties at each stage. After losing the structure of the cytoskeleton the cell shape becomes indefinite, and the cell can be deformed easily. These cells are mobile and can relocate easily. Reconstruction of MET needs re-establishing the intercellular connections.

could even be one of the key factors in EMT from bio-electromagnetism. As the process of cell fission begins, the water structure gradually becomes disordered around the cell [40], increasing the dielectric permeability of the water [41] and changing many transport properties [42]. This is probably connected with the cytoskeletal restructuring that takes place during EMT, with the fixed polarization of the cellular layer being gradually lost, making disorder by of the connected water layer. The increased permittivity caused by disorder further decreases cell-cell adhesion and may be additional support of the cell-division or even for the proliferation. From water ordering and polarization, the two phases of the transition are defined as the beta $(\beta$, collective, well polarized, ordered, low dielectric permittivity) and alpha ( $\alpha$, autonomic, not polarized, disordered, high dielectric permittivity) states [43]. Highly organized, developed living tissues are naturally built up from water structures in the $\beta$ state. This cellular fission is well controlled. One of the guiding factors is the water order, which regulates the interaction forces between the cells without considering actual chemical bonds.

The order-disorder transition of the aqueous electrolytes probably plays a role 


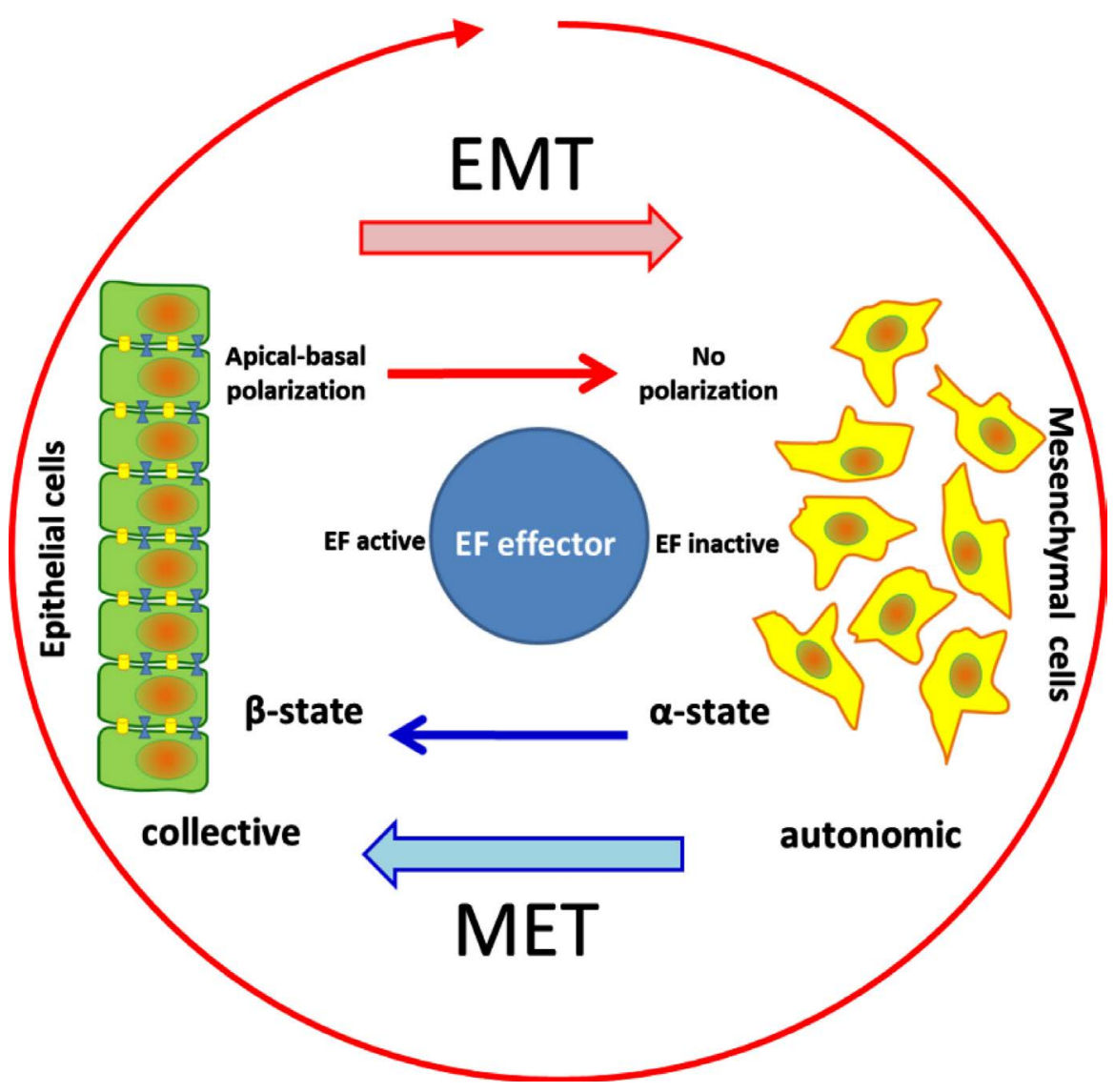

Figure 3. The role of EF as an effector in the EMT-MET pair of transitions.

in the $\alpha \rightarrow \beta$ transitions [44]. This ordering of the water states may promote the adherent bonds during the end-stage of MET. In the same way, the changes in the final stages of EMT are supported by the disordering of the surrounding water structures (Figure 3 ).

The cells in the healthy tissue are in cooperative interactions mostly run on mitochondria-driven metabolism, producing 36 ATPs from one glucose molecule in the rather complex Krebs cycle. When cellular cleavage starts, a larger energy flux is necessary to support the production of the daughter cells or simply to supply energy for the cellular transitions. The larger demand for energy could activate glycolytic energy production mainly under hypoxic conditions. Mesenchymal cancer cells are mostly hypoxic, and so exhibit a high rate of glycolysis [45]. In this simple process, only two ATPs are produced from one glucose molecule. The efficacy of this glycolysis is low but is compensated by the large flux of materials due to the very simple production process. The intensive energy flux of the fermentative metabolism increases the heat liberated in the cell, and thus the temperature gradient between the extra and intracellular compartments. The increasing temperature difference will eventually reach a critical threshold, at which the heat flow changes from conductive to convective [46]. The convective flow promotes ionic flow through the cellular membrane, increasing the membrane permeability to glucose and thus supporting the glycolytic way of meta- 
bolism together with changes in the intracellular circulation [47] [48].

An interesting relation between energy flux and cooperativity in vitro, the specific metabolic rate in the cultured cells was constant [49] in conditions when overall metabolic rate normalized to the actual mass. The reason for this is simple: when available nutrient levels are practically infinite due to the permanent concentration of nutrients for the culture, the efficacy of energy use is not an issue. High supportive energy flux makes the cells autonomic, placing them in the $\alpha$ state. However, when the cells are cooperative the scaling shows $(-1 / 4)$ exponent [47], which characterizes the general allometric scaling of the specific metabolic rate vs. mass [50] [51]. Consequently, the metabolic rate depends on collectivity; when cells are cooperative, the efficacy of energy consumption is better than in the case of autonomic cells. A low energy supply makes the cells cooperative and also sophisticated, being very efficient in power generation and environmental adaptation as well. A spectacular demonstration of this is shown by slime-mold cells [52]. In the case of "infinite" nutrient availability, the cells are autonomic. However, upon starvation of slime mold, some cells act as collecting centers, leading to the aggregation of cells, and enhancing the efficacy of energy use due to the limited availability of nutrients.

\section{Effect of EF}

There are two consequences of the separation of epithelial cells: the cytoskeleton collapses at least partially, and the intercellular bonds disintegrate (at least intercellular signaling is disrupted). In this way, cell migration is promoted, and a new kind of elongated, fusiform cellular arrangement could appear, allowing the formation of chain-like structures. Effectors promote this process. They free the cells and allow the reorganization of the cells (transforming them into the mesenchymal form). One of the effectors of this process is a form-factor that is responsible for morphogenesis: this is the electric field (EF) [53].

The epithelial and mesenchymal cells differ from a functional and phenotypic point of view. Epithelial cells form layers in which the cells interact closely and are well connected by specific transmembrane proteins, forming adherent connections and various junctions. The epithelial cells are polarized in the apical-basolateral direction, due to the special local distribution of cadherin and integrin molecules, and the surrounding ordered structure of aqueous electrolyte ( $\beta$ state). Epithelial cells may have some displacements in their neighborhood but strictly remain in the epithelial layer.

Mesenchymal cells do not form any ordered layer, do not show polarization and are not connected by adherins, integrins or junctions. They have a disordered aqueous electrolyte structure ( $\alpha$ state). Their fusiform shape, as well as the disordered electrolyte structure, supports their migration and the formation of chains. This shape ensures the heterogenic charge distribution on their membrane, which influences the chain orientation and ordering and supports their migration along the lines of EF. Hypoxic and necrotic cells liberate EMT effec- 
tors into the ECM, supporting the destruction of connections between the endothelial cells and collapsing the standard cytoskeletal network intracellularly.

Electromagnetic effects could initiate regeneration of the intercellular connection (transformation from $\alpha$-state to $\beta$-state). Experiments show the reestablishing of the E-cadherin $\beta$-catenin complex intercellular bonds [54], and downregulates the molecular factors for neoangiogenesis, [55].

The role of EF-induced biocurrents has been debated in biophysics for many years. The automatic biological charge transfers have an important role and might be a basic phenomenon of tissue repair [56] [57].

To clarify the role of EF as an effector of angiogenesis, we again apply the permanence principle, using the morphogenesis of epithelial cells to investigate in detail the processes connected to wound healing [58]. The inner side of the epithelial layer is positively charged, while the outside is negative. In the cornea (which has been studied in detail), this gradient, which is called the transcorneal potential difference (TPC), is caused by an active ion pump that produces an influx of positive $\mathrm{Na}^{+}$and $\mathrm{K}^{+}$ions and efflux of negative $\mathrm{Cl}$-ions. The value of the TPC is typically $40 \mathrm{mV}$. In the case of injury, the wound in the epithelium provides a short-cut, its potential tends to zero in this localization. However, in distance of $05-1 \mathrm{~mm}$, the original potential value could be measured, causing a certain gradient of EF. This EF-difference induces electric current directed to the wound (Figure 4). The current, powered by this process of the endogenous field-strength, is called injury-current, [59].

The injury current certainly plays a central role in wound healing [60]. Injury currents are physiological [61], and their typical value is around $100 \mu \mathrm{A} / \mathrm{cm}^{2}$ on the physiological potential-gradient drops $\sim 100 \mathrm{mV} / \mathrm{cm}$ and may be extended to $\mathrm{mm}$ distance from the wound [50]. This very low power (approx. $0.01 \mathrm{~mW} / \mathrm{g}$ ) does not increase the local temperature, [62], but can be measured using high-tech methods during the wound-healing process [63] [64] [65]. The EF in the tissue is oriented to the wounded area, and the current has a closed loop through the surface of the epithelium, with the current traveling from inside to the surface of the wound itself. This electrically controls the wound-healing process and persists as long as the wound exists. It regulates the orientation and the frequency of cell division [66], directs the cell migration to heal the wound [67].

\section{Discussion}

The endogenous EF that gives rise to the injury current is a primary morphogen, acting on the inner side of the epithelium. The physiological effects of the endogenous EF are shown in Figure 5.

During the wound-healing process, cells isolate themselves from others, breaking the adherent connections and junctions, while de-structuring of the cytoskeleton results in deformability and the possible formation of new shapes. The injury itself produces growth factors and compounds promoting cellular fission. This effect is intensive apart from the wound, where the injury-induced 


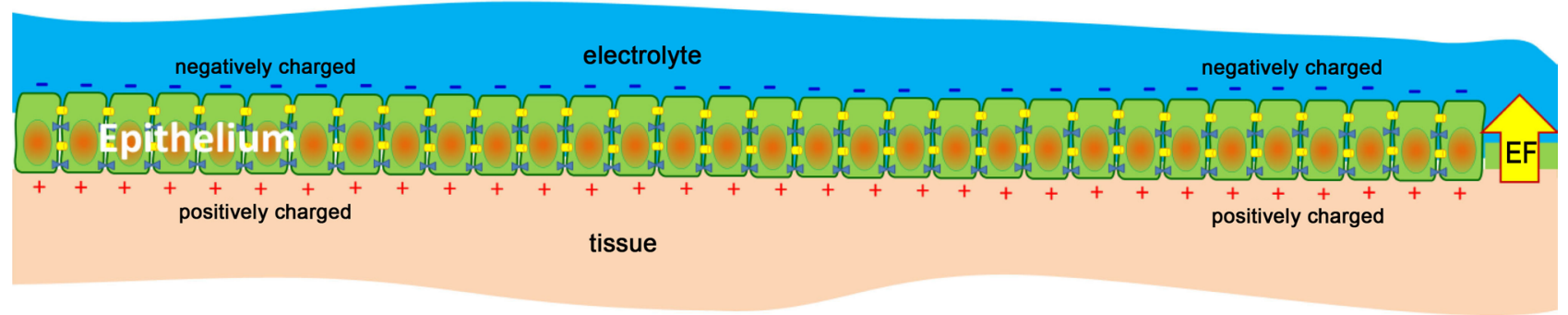

(a)

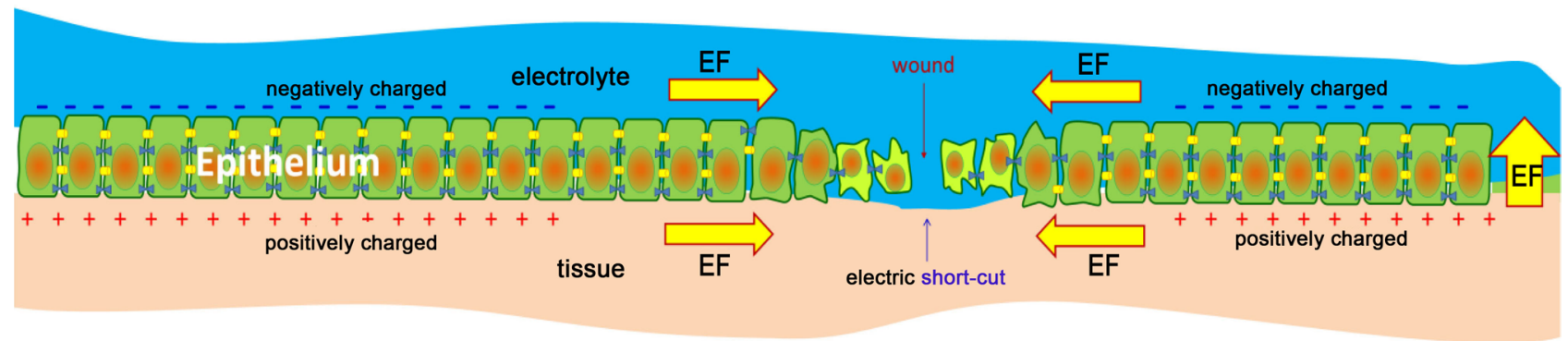

(b)

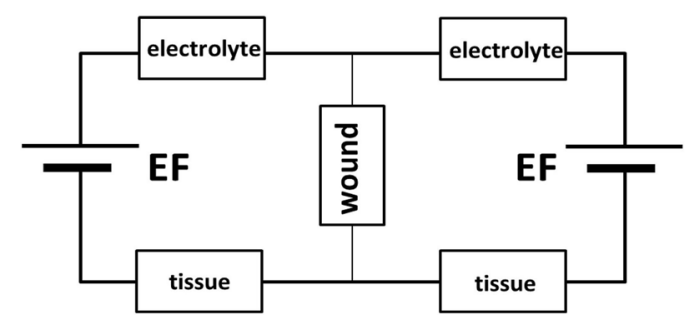

(c)

Figure 4. Generation of the endogenous EF and injury current. (a) No current, polarization is constant. (b) The wound provides a short-cut, through which the current flows. (c) A circuit picture of the injury current. When the injury is healed, there is no longer a shortcut, so the current disappears, and the situation shown in (a) is re-established.

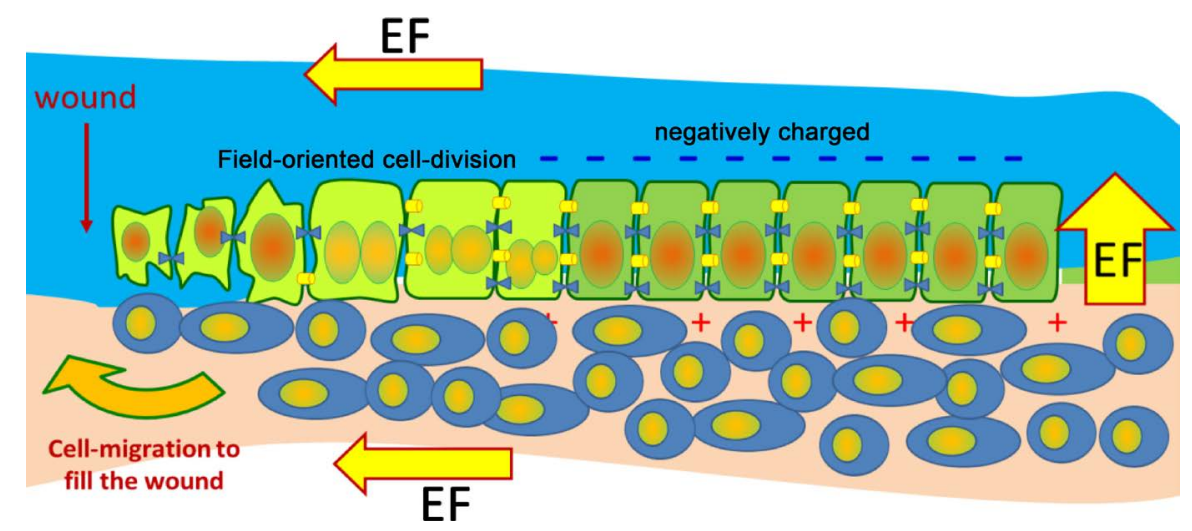

Figure 5. Physiological processes are driven by the endogenous EF. Cell migration during wound healing induced by the injury current is well documented.

electric field is low. Together with the new cells, the wound shows directional proliferation (centripetal migration [68], regulated from a relatively large distance, $0.5-1 \mathrm{~mm}$ ). The steps in wound healing are as follows:

- Cells migrate from the distant (healthy) domains toward the wound (Figure 
6);

- The axis of proliferation is oriented at the verge of the wound. Proliferation declines gradually with distance from the wound;

- The current is proportional to the EF-induced by the injury;

- The speed of wound healing is accelerated by a higher EF;

- EF controls cell proliferation at the wound. It orients the cells and the development of the nerve and transport networks;

- EF changes the distribution of the receptors of growth factors along the cell membranes;

- The normalized on control of potential difference could be altered by drugs.

At the start of angiogenesis new cells develop, and these are more negatively charged than their older counterparts; furthermore, their supply of nutrients and oxygen is not yet organized. The local hypoxia induces VEGF, which is supported by the gradient of EF caused by the negative newborn cells. In this case, the new cells in meaning to generate EF and EF-induced current play the same role as a wound. The old vessel wall starts rearranging in isolated areas, and the free wound helps build up the new vessel network by the injury current. Vessel forming may lead to bifurcative branching, for what the mathematical model exists [69]. We state that the morphogenesis needs EF, as it controls the orientation of cell fission, and regulates the direction of cell migration as well as the movement of compounds (like growth factors) towards the site of action.

Based on the permanence principle we hypothesize that the EF is caused by the cleavage and appearance of the new cells (Figure 6).

Experimental proof of the oriented cell division of epithelial cells has been

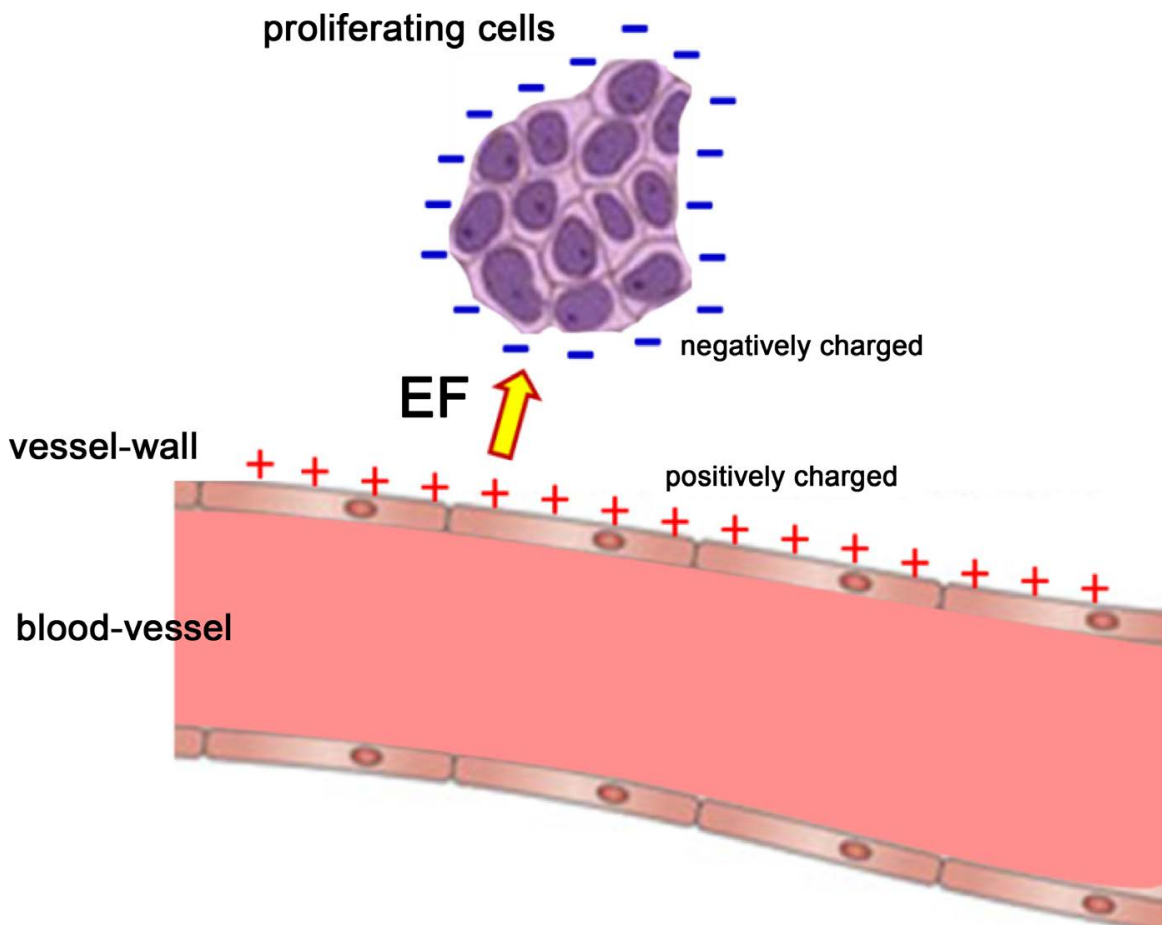

Figure 6. EF robustly formed when a group of newborn cells appears in the tissue. 
presented [70]. The endogenous EF organizes the dipole molecules and could increase the lipids and associated receptors of growth factors on the negative pole. When the ends of astral microtubules bind to the transmembrane proteins, the $\mathrm{EF}$ orients the spindle microtubules intracellularly.

Possible mechanisms of cell migration are also connected to the effect of EF. Without EF the cells would have a negative membrane potential and an equilibrated distribution of charges (dipoles) in the membrane. When EF appears the side of the cell nearest to the positive pole hyperpolarizes. This polarization effect modifies the $\mathrm{Ca}^{2+}$ transport from the ECM. When neglecting the voltage-gated effects, the diffusion will form a larger concentration of $\mathrm{Ca}^{2+}$ in the intracellular electrolyte on the positive pole of the cell than on the negative side. Due to this misbalance, the positive side of the cell undergoes a contraction, while the negative side dilates at the same time. This causes a displacement (migration) of the cell in the positive-to-negative direction of the EF [71].

In the case of active voltage-gated ionic channels are considered the ion concentration on the positive side of the cytoplasm increases by diffusion. On the negative electric pole of the cell, the activity of voltage-gated channels will be intensified by the depolarization of the EF. These effects both lead to contraction of the cell. The direction of the displacement is determined by the size of the contractions. The action of voltage-gated ion channels is more efficient than diffusion, causing migration in the opposite direction than would occur without voltage-gated channels. Migration may involve individual cells and clusters of cells too.

Oriented cell fission and migration are highest at the edge of the wound, while proliferation is maximal when the EF is zero. This means that wound healing results from centripetal cell migration towards the wound. More precisely, the process of migration delivers the cells, but the oriented cell division makes it ordered and forms the tissue structure.

The above considerations of the permanence principle in the case of angiogenesis suggest that the endothelial cells migrate from a distance guided by the old vessel wall towards the negative newborn cells, where the large EF accelerated migration and oriented cellular fission. This may explain how the diameter of the blood vessel becomes smaller and the tube narrower, forming a capillary in the distance. In the next step of angiogenesis, the cells leave its cycle in a hostile environment to survive and build up a physiological capillary structure. In the final step of angiogenesis, the appropriate ECM is built up, cellular connections are formed, and other cells (like smooth muscle cells) complete the complex structure of the vessel tissue. Angiopoietin molecules play a role in this stage of development, by uniting the new capillary with the existing one, forming a physiologically functional structure.

The effectors forming the cellular connections are mandatory in the angiogenic process. These connections probably start with E-cadherin, which initializes the next steps via EF construction. The electrochemistry of the cytoskeleton 
polymerization may be as follows: The positive side of the cell is hyperpolarized, increasing the $\mathrm{Ca}^{2+}$ influx into the cell by diffusion. On the negative side, the voltage-gated channels pump more $\mathrm{Ca}^{2+}$ into the cell. In this way the $\mathrm{Ca}^{2+}$ concentration in the cell gradually increases, activating the rho GTPases:

$\mathrm{Ca}^{2+} \rightarrow$ adenylate cyclase $\rightarrow$ cAMP/PKA $\rightarrow$ rho GTPases $\rightarrow$ cytoskeleton

which is mandatory in the building of the cytoskeleton [50].

It is clear that the process is the same when the $\mathrm{EF}$ is in the opposite direction; consequently, the process takes place in the alternating field too, although the direction of migration would be indefinite.

Via this process, the cells finally build the vessel wall, and gradually build up their final communication channels too.

The final stage of angiogenesis comprises remodeling when the complete network is optimized for its task. The un-used capillaries dissolve, and anastomoses appear between the capillaries.

All the above considerations are entirely applicable to the development of malignancy. The malignant tissue has a specific gradient in potential with its healthy neighborhood [62] [71], and this acts to promote and direct cancer cell migration [72]. There are arguments on the cancerous process as a wound repair [73]. The bio-system falsely recognizes the tumor as a wound and stimulates its environment to heal the irregularity (that is, to produce cells to heal the wound). This false wound-healing mechanism is actively supported by the actual injury currents caused by the potential gradients.

Cancer treatment has been developed [74] [75] based on the concept of "biologically closed electric circuits" (BCEC) [76] [77]. This type of method involves applying an external electric field to generate currents, and has been found effective against cancer [78] [79] [80]. Wound healing by external EF stimulation has also been reported [81].

Dielectric polarization depends on the gradients of permittivity and the natural logarithm of conductivity. Due to the weak dependence on conductivity, let us consider the permittivity gradient as a linear function:

$$
\bar{E} \cdot \Delta=\rho
$$

where $\Delta$ is the gradient of permittivity $(\Delta=\operatorname{grad}(\varepsilon)), \mathrm{E}$ is the electric field, and $\rho$ is the density of charge in the target. For the easiest geometry let us consider a disc-shaped skin cancer (surface tumor) with full cylindrical symmetry. Given its higher permittivity and negative charge compared to its healthy neighbors, the distribution of the physical parameters shown in Figure 7 is realistic.

The geometry of the tumor involves the maximal value of the space charge, and $E$ and $\Delta=\operatorname{grad}(\varepsilon)$ are parallel enough to calculate the product of their absolute values. The permittivity gradient is opposite to the field-strength vector, due to the negative charge of cancer. To compensate the negative space charge, the field restricts the electric current to the cancer disc, starting an injury current between the cancerous and healthy parts. This current could dedifferentiate 
(a)

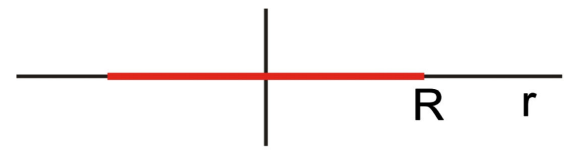

(b)

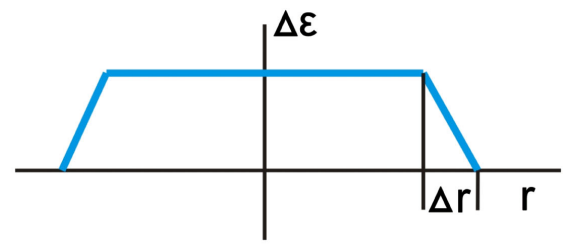

(c)

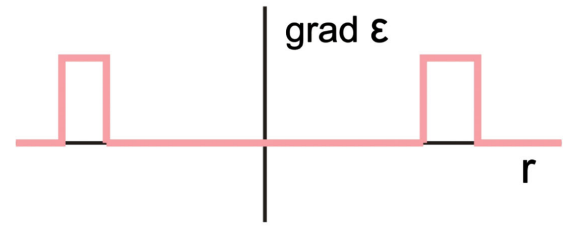

(d)

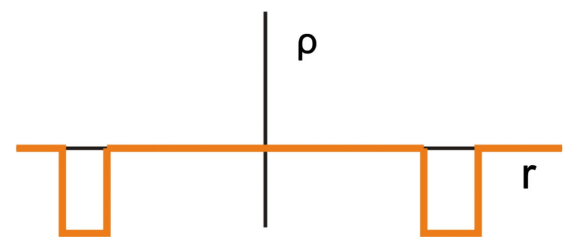

Figure 7. Distribution of relevant physical parameters in the disc-shaped tumor ( $\varepsilon$ is the dielectric permittivity, $\rho$ is the charge density). (a) The radius of the disc is R. (b) The change in the gradient effect widens this distance, by the modification or the dielectric permittivity in a $\Delta \mathrm{r}$-range. (c) The gradient of permittivity differs from zero only in the transition zone, (d) where the charge density is also not zero.

healthy cells into multipotent ones, which could become autonomic and redifferentiate into cancerous cells. This mechanism creates "precancerous cells" [82] [83]. Injury current is not able to compensate the space charge. Due to the high metabolic rate of the tumor, the cancerous cells are in a state of permanent division and produce a negative space charge. The natural mechanisms of the bio-system are not able to block the cancerous growth after a certain size. Consequently, artificial intervention to deliver positive charges to compensate could be helpful. For this, the necessary electric field strength should be parallel with the gradient of permittivity.

In cancerous proliferation, the massive numbers of newly produced cells create a definite EF. The injury current becomes permanent. The simplest reason for this is the grouping of the newborn cells, which have no possibility of neutralizing themselves to the potential of the natural surroundings. (The new cells are charged more negatively than the cells in the normal host tissue.) It can be hypothesized that as a constrained current the injury current depolarizes the cell membrane. The depolarization of the cell membrane is associated with proliferation [84] [85], and therefore could induce cell division and wound healing.

\section{Conclusion}

We argue that the process of angiogenesis is guided by the EF, which itself arises due to the polarization and proliferation of cells. By analogy with the epitheli- 
al-mesenchymal-transformation, we have reached conclusions about the endothelial-mesenchymal-transformation. The analogy shows a perfect fit with the available literature, so we can conclude that electric field and its effect on charge distribution have a role in endothelial-mesenchymal-transformation and consequently in the neoangiogenesis as well.

\section{Acknowledgements}

This work was supported by the Hungarian Competitiveness and Excellence Programme Grant (NVKP_16-1-2016-0042).

\section{References}

[1] Patan, S. (2004) Vasculogenesis and Angiogenesis. Cancer Treatment and Research, 117, 3-32. https://doi.org/10.1007/978-1-4419-8871-3_1

[2] Greaves, N.S., Ashcroft, K.J., Baguneid, M., et al. (2013) Current Understanding of Molecular and Cellular Mechanisms in Fibroplasia and Angiogenesis during Acute Wound Healing. Journal of Dermatological Science, 72, 206-217. https://doi.org/10.1016/j.jdermsci.2013.07.008

[3] Prior, B.M., Yang, H.T. and Terjung, R.L. (2004) What Makes Vessels Grow with Exercise Training? Journal of Applied Physiology, 97, 1119-1128. https://doi.org/10.1152/japplphysiol.00035.2004

[4] Adams, R.H. and Alitalo, K. (2007) Molecular Regulation of Angiogenesis and Lymphangiogenesis. Nature Reviews Molecular Cell Biology, 8, 464-478. https://doi.org/10.1038/nrm2183

[5] Algire, G.H. (1945) Vascular Reactions of Normal and Malignant Tissues in Vivo. I. Vascular Reactions of Mice to Wounds and to Normal and Neoplastic Transplants. Journal of the National Cancer Institute, 6, 73-85. https://doi.org/10.1093/jnci/6.1.73

[6] Hanahan, D. and Weinberg, R.A. (2000) The Hallmarks of Cancer. Cell, 100, 57-70. https://doi.org/10.1016/S0092-8674(00)81683-9

[7] Folkman, J. (1971) Tumor Angiogenesis: Therapeutic Implications. The New England Journal of Medicine, 285, 1182-1186. https://doi.org/10.1056/NEJM197111182852108

[8] Folkman, J. (1984) Angiogenesis. In: Jaffe, E.A., Ed., Biology of Endothelial Cells, Nijhoff, Boston, 412-428. https://doi.org/10.1007/978-1-4613-2825-4_42

[9] Markwald, R.R., Fitzharris, T.P. and Smith, W.N. (1975) Structural Analysis of Endocardial Cytodifferentiation. Developmental Biology, 42, 160-180. https://doi.org/10.1016/0012-1606(75)90321-8

[10] Markwald, R.R., Fitzharris, T.P. and Manasek, F.J. (1977) Structural Development of Endocardial Cushions. The American Journal of Anatomy, 148, 85-119. https://doi.org/10.1002/aja.1001480108

[11] Zeisberg, E.M., Potenta, S., Xie, L., et al. (2007) Discovery of Endothelial to Mesenchymal Transition as a Source for Carcinoma-Associated Fibroblasts. Cancer Research, 67, 10123-10128. https://doi.org/10.1158/0008-5472.CAN-07-3127

[12] Zeisberg, E.M., Tarnavski, O., Zeisberg, M., et al. (2007) Endothelial-to-Mesenchymal Transition Contributes to Cardiac Fibrosis. Nature Medicine, 13, 952-961. https://doi.org/10.1038/nm1613

[13] Kalluri, R. and Zeisberg, M. (2006) Fibroblasts in Cancer. Nature Reviews Cancer, 
6, 392-401. https://doi.org/10.1038/nrc1877

[14] Gerhardt, H., Golding, M., Fruttiger, M., et al. (2003) VEGF Guides Angiogenic Sprouting Utilizing Endothelial Tip Cell Filopodia. The Journal of Cell Biology, 161, 1163-1177. https://doi.org/10.1083/jcb.200302047

[15] Armulik, A., Abramsson, A. and Betsholtz, C. (2005) Endothelial/Pericyte Interactions. Circulation Research, 97, 512-523. https://doi.org/10.1161/01.RES.0000182903.16652.d7

[16] Mancardi, D., Varetto, G., Bucci, E., et al. (2008) Fractal Parameters and Vascular Networks: Facts \& Artifacts. Theoretical Biology and Medical Modelling, 5, 1-8.

[17] Xu, J., Vilanova, G. and Gomez, H. (2016) A Mathematical Model Coupling Tumor Growth and Angiogenesis. PLoS ONE, 11, e0149422.

https://doi.org/10.1371/journal.pone.0149422

[18] Potenta, S., Zeisberg, E. and Kalluri, R. (2008) The Role of Endothelial-to-Mesenchymal Transition in Cancer Progression. British Journal of Cancer, 99, 1375-1379. https://doi.org/10.1038/sj.bjc.6604662

[19] Batlle, E., Sancho, E., Franci, C., et al. (2000) The Transcription Factor Snail Is a Repressor of E-Cadherin Gene Expression in Epithelial Tumour Cells. Nature Cell Biology, 2, 84-89. https://doi.org/10.1038/35000034

[20] Cano, A., Perez-Moreno, M.A., Rodrigo, I., et al. (2000) The Transcription Factor Snail Controls Epithelial-Mesenchymal Transitions by Repressing E-Cadherin Expression. Nature Cell Biology, 2, 76-83. https://doi.org/10.1038/35000025

[21] Zavadil, J. and Bottinger, E.P. (2005) TGF-Beta and Epithelial-to-Mesenchymal Transitions. Oncogene, 24, 5764-5774. https://doi.org/10.1038/sj.onc.1208927

[22] Tse, J. and Kalluri, R. (2007) Mechanisms of Metastasis: Epithelial-to-Mesenchymal Transition and Contribution of Tumor Microenvironment. Journal of Cellular Biochemistry, 101, 816-829. https://doi.org/10.1002/jcb.21215

[23] Kovacic, J.C., Mercader, N., Torres, M., et al. (2012) Epithelial-to-Mesenchymal and Endothelial-to-Mesenchymal Transition from Cardiovascular Development to Disease. Circulation, 125, 1795-1808. https://doi.org/10.1161/CIRCULATIONAHA.111.040352

[24] Gurzu, S., Turdean, S., Kovecsi, A., Contac, A.O., et al. (2015) Epithelial-Mesenchymal, Mesenchymal-Epithelial, and Endothelial-Mesenchymal Transitions in Malignant Tumors: An Update. World Journal of Clinical Cases, 3, 393-404. https://doi.org/10.12998/wjcc.v3.i5.393

[25] Piera-Velazquez, S., Zhaodong, L. and Jimenez, S.A. (2011) Role of Endothelial-Mesenchymal Transition (EndoMT) in the Pathogenesis of Fibrotic Disorders. The American Journal of Pathology, 179, 1074-1081. https://doi.org/10.1016/j.ajpath.2011.06.001

[26] Bryant, D.M. and Mostov, K.E. (2008) From Cells to Organs: Building Polarized Tissue. Nature Reviews Molecular Cell Biology, 9, 887-901. https://doi.org/10.1038/nrm2523

[27] Cope, F.W. (1969) Nuclear Magnetic Resonance Evidence using D2O for Structured Water in Muscle and Brain. Biophysical Journal, 9, 303-319. https://doi.org/10.1016/S0006-3495(69)86388-5

[28] Hazlewood, C.F., Chang, D.C., Medina, D., et al. (1972) Distinction between the Preneoplastic and Neoplastic State of Murine Mammary Glands. Proceedings of the National Academy of Sciences, 69, 1478-1480.

https://doi.org/10.1073/pnas.69.6.1478

[29] Damadian, R. (1971) Tumor Detection by Nuclear Magnetic Resonance. Science, 
171, 1151-1153. https://doi.org/10.1126/science.171.3976.1151

[30] Thiery, J.P. and Sleeman, J.P. (2006) Complex Networks Orchestrate Epithelialmesenchymal Transitions. Nature Reviews Molecular Cell Biology, 7, 131-142. https://doi.org/10.1038/nrm1835

[31] Liang, X., Gomez, G.A. and Yap, A.S. (2015) Current Perspectives on Cadherin-Cytoskeleton Interactions and Dynamics. Dove Medical Press, 7, 11-24.

[32] Tsai, J.H. and Yang, J. (2013) Epithelial-Mesenchymal Plasticity in Carcinoma Metastasis. Genes \& Development, 27, 2192-2206. https://doi.org/10.1101/gad.225334.113

[33] Wai, L.T. and Weinber, R.A. (2013) The Epigenetics of Epithelial-Mesenchymal Plasticity in Cancer. Nature Medicine, 19, 1438-1449. https://doi.org/10.1038/nm.3336

[34] Wong, I.Y., Javaid, S., Wong, E.A., et al. (2014) Collective and Individual Migration Following the Epithelial-Mesenchymal Transition. Nature Materials, 13, 1063-1071. https://doi.org/10.1038/nmat4062

[35] Hegyi, G., Vincze, Gy. and Szasz, A. (2012) On the Dynamic Equilibrium in Homeostasis. Open Journal of Biophysics, 2, 64-71. https://doi.org/10.4236/ojbiphy.2012.23009

[36] Tyler (2015) Understanding Mesenchymal to Epithelial Cell Transition May Be Key for Neo-Growth Plates. Natural Height Growth. http://www.naturalheightgrowth.com/2015/11/11/understandingmesenchymalendo thelialcelltransitionmaykeyneogrowthplates/

[37] Thiery, J.P., Acloque, H., Huang, R.Y., et al. (2009) Epithelial-Mesenchymal Transitions in Development and Disease. Cell, 139, 871-890. https://doi.org/10.1016/j.cell.2009.11.007

[38] Hugo, H., Ackland, M.L., Blick, T., et al. (2007) Epithelial-Mesenchymal and Mesenchymal-Epithelial Transitions in Carcinoma Progression. Journal of Cellular Physiology, 213, 374-383. https://doi.org/10.1002/jcp.21223

[39] Szentgyorgyi, A. (1998) Electronic Biology and Cancer. Marcel Dekker, New York.

[40] Szentgyorgyi, A. (1957) Bioenergetica. Academic Press, New York.

[41] Buchner, R., Barthel, J. and Stauber, J. (1999) The Dielectric Relaxation of Water between $0{ }^{\circ} \mathrm{C}$ and $35{ }^{\circ} \mathrm{C}$. Chemical Physics Letters, 306, 57-63. https://doi.org/10.1016/S0009-2614(99)00455-8

[42] Gascoyne, P.R., Pethig, R. and Szentgyorgyi, A. (1981) Water Structure-Dependent Charge Transport in Proteins (Protons/Electrons/Charge Transfer/Dielectric Dispersion). Proceedings of the National Academy of Sciences, 78, 261-265.

[43] Szentgyorgyi, A. (1978) The Living State and Cancer. Marcel Dekker Inc., New York.

[44] Szentgyorgyi, A. (1968) Bioelectronics: A Study on Cellular Regulations, Defense and Cancer. Acad. Press, New York, London.

[45] Shiraishi, T., Verdone, J.E., Huang, J., et al. (2014) Glycolysis Is the Primary Bioenergetic Pathway for Cell Motility and Cytoskeletal Remodeling in Human Prostate and Breast Cancer Cells. Oncotarget, 6, 130-143.

[46] Kurakin, A. (2009) Scale-Free Flow of Life: On the Biology, Economics, and Physics of the Cell. Theoretical Biology and Medical Modelling, 6, 6 .

[47] Hochachka, P.W. (1999) The Metabolic Implications of Intracellular Circulation. Proceedings of the National Academy of Sciences, 96, 12233-12239. 
https://doi.org/10.1073/pnas.96.22.12233

[48] Coulson, R.A. (1986) Metabolic Rate and the Flow Theory: A Study in Chemical Engineering. Comparative Biochemistry and Physiology Part A, 84, 217-229. https://doi.org/10.1016/0300-9629(86)90607-9

[49] Brown, M.F., Gratton, T.P. and Stuart, J.A. (2007) Metabolic Rate Does Not Scale with Body Mass in Cultured Mammalian Cells. American Journal of Physiology. Regulatory, Integrative and Comparative Physiology, 292, 2115-2121. https://doi.org/10.1152/ajpregu.00568.2006

[50] Kleiber, M. (1947) Body Size and Metabolic Rate. Physiological Reviews, 27, 511-541. https://doi.org/10.1152/physrev.1947.27.4.511

[51] West, G.B., Brown, J.H. and Enquist, B.J. (1999) The Fourth Dimension of Life: Fractal Geometry and Allometric Scaling of Organisms. Science, 284, 1677-1679. https://doi.org/10.1126/science.284.5420.1677

[52] Bonner, J.T. (1967) The Cellular Slime Moulds. 2nd Edition, Princeton University Press, Princeton.

[53] Martin-Belmonte, F. and Mostov, K. (2008) Regulation of Cell Polarity during Epithelial Morphogenesis. Current Opinion in Cell Biology, 20, 227-234. https://doi.org/10.1016/j.ceb.2008.01.001

[54] Yang, K.L., Huang, C.C., Chi, M.S., et al. (2016) In Vitro Comparison of Conventional Hyperthermia and Modulated Electro-Hyperthermia. Oncotarget, 7, 84082-84092.

[55] Kim, E.H., Song, H.S., Seung S.H., et al. (2016) Tumor Treating Fields Inhibit Glioblastoma Cell Migration, Invasion and Angiogenesis. Oncotarget, 7, 65125-65136.

[56] Becker, R.O. and Selden, G. (1985) The Body Electric. Morrow, New York.

[57] Becker, R.O. (1990) Cross Currents. Jeremy P Tarcher Inc., Los Angeles.

[58] McCaig, C.D., Rajnicek, A.M., Song, B., et al. (2005) Controlling Cell Behaviour Electrically: Current Views and Future Potential. Physiological Reviews, 85, 943-978. https://doi.org/10.1152/physrev.00020.2004

[59] Rosch, P.J. and Markov, M.S. (2004) Bioelectromagnetic Medicine. Marcell Decker Inc., New York.

[60] Reid, B., McCaig, C.D., Zhao, M., et al. (2005) Wound Healing in Rat Cornea: The Role of Electric Currents. FASEB J, 19, 379-386.

https://doi.org/10.1096/fj.04-2325com

[61] Barker, A.T., Jaffe, L.F. and Vanable, J.W. (1982) The Glabrous Epidermis of Cavies Contains a Powerful Battery. American Journal of Physiology, 242, 358-366.

[62] Song, B., Zhao, M., Forrester, J., et al. (2004) Nerve Regeneration and Wound Healing Are Stimulated and Directed by an Endogenous Electrical Field in Vivo. Journal of Cell Science, 117, 4681-4690. https://doi.org/10.1242/jcs.01341

[63] Carbon, M., Wübbeler, G., Mackert, B.M., et al. (2004) Non-Invasive Magnetic Detection of Human Injury Currents. Clinical Neurophysiology, 115, 1027-1032. https://doi.org/10.1016/j.clinph.2003.12.035

[64] Reid, B., Nuccitelli, R. and Zhao, M. (2007) Non-Invasive Measurement of Bioelectric Currents with a Vibrating Probe. Nature Protocols, 2, 661-669. https://doi.org/10.1038/nprot.2007.91

[65] Mackert, B.M., Mackert, J., Wübbeler, G., et al. (1999) Magnetometry of Injury Currents from Human Nerve and Muscle Specimens using Superconducting 
Quantum Interferences Devices. Neuroscience Letters, 262, 163-166. https://doi.org/10.1016/S0304-3940(99)00067-1

[66] Song, B., Zhao, M., Forrester, J.V., et al. (2002) Electrical Cues Regulate the Orientation and Frequency of Cell Division and the Rate of Wound Healing in Vivo. PNAS, 99, 13577-13582. https://doi.org/10.1073/pnas.202235299

[67] Zhao, M. (2009) Electrical Fields in Wound Healing-An Overriding Signal That Directs Cell Migration. Seminars in Cell and Developmental Biology, 20, 674-682. https://doi.org/10.1016/j.semcdb.2008.12.009

[68] Buck, R.C. (1985) Measurement of Centripetal Migration of Normal Corneal Epithelial Cells in the Mouse. Investigative Ophthalmology \& Visual Science, 26, 1296-1299.

[69] Adler, P.M. (1992) Porous Media Geometry and Transport. Butterworth-Heinemann, Boston, London, Oxford.

[70] Zhao, M., Forrester, J.V. and McCaig, C.D. (1999) A Small, Physiological Electric Field Orients Cell Division. Proceedings of the National Academy of Sciences, 96, 4942-4946.

[71] Mycielska, M.E. and Djamgoz, M.B.A. (2004) Cellular Mechanisms of Direct-Current Electric Field Effects: Galvanotaxis and Metastatic Disease. Journal of Cell Science, 117, 1631-1639. https://doi.org/10.1242/jcs.01125

[72] Pu, J., McCaig, C.D., Cao, L., et al. (2007) EGF Receptor Signalling Is Essential for Electric-Field-Directed Migration of Breast Cancer Cells. Journal of Cell Science, 120, 3395-3403. https://doi.org/10.1242/jcs.002774

[73] Meng, X. and Riordan, N.H. (2006) Cancer Is a Functional Repair Tissue. Medical Hypotheses, 66, 486-490. https://doi.org/10.1016/j.mehy.2005.09.041

[74] Nordenström, B.E.W. (1978) Preliminary Clinical Trials of Electrophoretic Ionization in the Treatment of Malignant Tumors. IRCS Medical Science, 6, 537-540.

[75] Nordenström, B.E.W. (1985) Electrochemical Treatment of Cancer. Annales De Radiologie, 28, 128129.

[76] Nordenstrom, B.W.E. (1983) Biologically Closed Electric Circuits: Clinical Experimental and Theoretical Evidence for an Additional Circulatory System. Nordic Medical Publications, Stockholm.

[77] Nordenstrom, B.W.E. (1998) Exploring BCEC-Systems, (Biologically Closed Electric Circuits). Nordic Medical Publications, Stockholm.

[78] Watson, B.W. (1991) Reappraisal: The Treatment of Tumors with Direct Electric Current. Medical Science Research, 19, 103-105.

[79] Samuelsson, L., Jonsson, L. and Stahl, E. (1983) Percutaneous Treatment of Pulmonary Tumors by Electrolysis. Radiologie, 23, 284-287.

[80] Miklavcic, D., Sersa, G., Kryzanowski, M., et al. (1993) Tumor Treatment by Direct Electric Current, Tumor Temperature and pH, Electrode Materials and Configuration. Bioelectrochemistry and Bioenergetics, 30, 209-220. https://doi.org/10.1016/0302-4598(93)80080-E

[81] Ud-Din, S., Sebastian, A., Giddings, P., et al. (2015) Angiogenesis Is Induced and Wound Size Is Reduced by Electrical Stimulation in an Acute Wound Healing Model in Human Skin. PLoS ONE, 10, e0124502. https://doi.org/10.1371/journal.pone.0124502

[82] Loewenstein, W.R. and Kanno, Y. (1967) Intercellular Communication and Tissue Growth. The Journal of Cell Biology, 33, 225-234.

https://doi.org/10.1083/jcb.33.2.225 
[83] Loewenstein, W.R. (1999) The Touchstone of Life, Molecular Information, Cell Communication and the Foundations of the Life. Oxford University Press, Oxford, New York, 298-304.

[84] James, A.M., Ambrose, E.J. and Lowick, J.H.B. (1956) Differences between the Electrical Charge Carried by Normal and Homologous Tumor Cells. Nature, 177, 576-577. https://doi.org/10.1038/177576a0

[85] Binggeli, R. and Weinstein, R.C. (1986) Membrane Potentials and Sodium Channels: Hypotheses for Growth Regulation and Cancer Formation Based on Changes in Sodium Channels and Gap Junctions. Journal of Theoretical Biology, 123, 377-401. https://doi.org/10.1016/S0022-5193(86)80209-0 